\title{
Connection between soft and hard probes of small collision sys- tems at RHIC and LHC
}

\author{
Dennis V. Perepelitsa ${ }^{1, \star}$ \\ ${ }^{1}$ University of Colorado Boulder, 2000 Colorado Ave, Boulder, CO 80309 USA
}

\begin{abstract}
.
The expected connections between signatures in the soft and hard sectors of small collision systems, and the status of experimental attempts to identify them, are discussed. These proceedings summarize the talk as given at the International Symposium on MultiParticle Dynamics in September 2019 in Santa Fe, NM (ISMD19). As such, the choice of content and focus are selective and not intended to be comprehensive.
\end{abstract}

\section{Introduction}

A significant open challenge in the study of QCD systems and their interactions is to understand the nature of the system formed in high-multiplicity proton-proton $(p p)$ or proton-nucleus $(p+\mathrm{A})$ collisions $[1,2]$ at the Relativistic Heavy Ion Collider (RHIC) and the Large Hadron Collider (LHC).

Analyses of the correlations between final-state particles in these systems reveal that they behave collectively, with their yields at fixed transverse momentum $\left(p_{\mathrm{T}}\right)$ following common, preferential orientations in the transverse plane. These patterns are popularly represented as a series of $n$-th order azimuthal anisotropy coefficients, $v_{n}$. In nucleus-nucleus $(\mathrm{A}+\mathrm{A})$ collisions, this bulk particle behavior can be well described using theoretical frameworks based on a viscous hydrodynamical description of final-state interactions in the Quark-Gluon Plasma (QGP) medium. Remarkably, these models are also able to describe the $v_{n}$ values as a function of $p_{\mathrm{T}}$ across $p p$ and $p+\mathrm{A}$ collisions, in a common paradigm with A+A collisions [3].

This picture is strengthened by a large body of corroborating data, including but not limited to: the "projectile scan" program at RHIC [4], in which $p+\mathrm{Au}, d+\mathrm{Au}$ and ${ }^{3} \mathrm{He}+\mathrm{Au}$ collisions are used to produce systems with differing initial-state geometric anisotropies $\varepsilon_{2,3}$ which are then observed to be translated into corresponding differences in the measured $v_{2,3}$ (shown in Fig. 1); the observation of hadron mass ordering in the $v_{n}$ coefficients [6], as expected from motion in a common velocity field; and measurements of multi-particle correlations which verify that the observed anisotropies are truly event-wide phenomena affecting all particles [7].

\section{Expectations for the hard sector}

For measurements of particles with $p_{\mathrm{T}} \lesssim 3 \mathrm{GeV}$ (the so-called "soft sector"), the paradigm described above with final-state interactions between strongly-interacting particles is dominant and widely supported by data. The purpose of these proceedings is to discuss what, given this paradigm, may be

^e-mail: dvp@ colorado.edu 

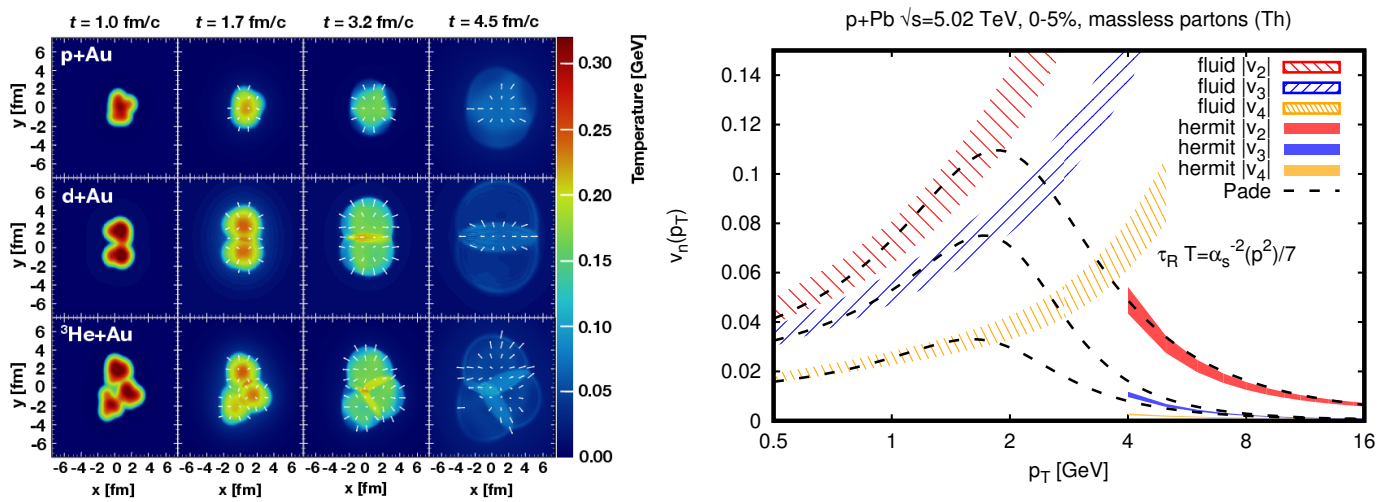

Figure 1. Left: Demonstration of hydrodynamic expansion of QGP medium in small collision systems, as part of the RHIC "projectile scan," from Ref. [4]. Right: Calculated $v_{2,3,4}$ values in central $p+\mathrm{Pb}$ collisions at low and high $p_{\mathrm{T}}$, from Ref. [5].

expected in the so-called "hard sector", where particle production is dominated by the fragmentation of high- $p_{\mathrm{T}}$ partons that emerge as jets. Rather than being treated as two unrelated phenomena, one should strive to understand the two behaviors together, since ultimately they are taking place concurrently in the same physical system.

In $\mathrm{A}+\mathrm{A}$ collisions, the dominant behaviors in soft and hard sectors are indirectly connected through the common relationship of each to the underlying geometry of the collision: low- $p_{\mathrm{T}}$ particles acquire an azimuthal anisotropy from the larger pressure gradient along the minor axis of the elliptic overlap region during the hydrodynamic expansion, while high- $p_{\mathrm{T}}$ particles acquire it from azimuthally-differential energy loss. Thus both low- $p_{\mathrm{T}}$ and high- $p_{\mathrm{T}}$ particle pair yields follow a $\cos (2 \Delta \phi)$ modulation. In fact, attempting to simultaneously understand differential energy loss and hydrodynamic flow in A+A collisions has spurred important theoretical developments [8].

One direct calculation of what may be expected at high- $p_{\mathrm{T}}$ in $p+\mathrm{A}$ collisions is performed in Ref. [5], and is summarized in Fig. 1. The calculation uses a common hydrodynamically evolving background and determines the $v_{2,3,4}$ for particles in many-scattering and few-scattering limits at low and high $p_{\mathrm{T}}$, respectively. This calculation indicates that appreciable $v_{2}$ values on the order of $2 \%$ for $p_{\mathrm{T}}>10 \mathrm{GeV}$ particles should inescapably accompany a non-trivial $v_{2}$ at low $p_{\mathrm{T}}$. However, in most models [9] this necessarily results in a significant effect in the nuclear modification factor, $R_{p \mathrm{~A}}$, which is not borne out by data: a large set of jet and charged-particle measurements have indicated that in minimum-bias $p+\mathrm{A}$ collisions, there is no detectable modification.

In the first $p+\mathrm{A}$ data taken in 2013 at the LHC, the total event statistics prohibited a detailed study of this high $p_{\mathrm{T}}$ region, even in ultra high-multiplicity events enhanced by special triggers. Nevertheless, the early results in Ref. [12] suggested that $v_{2}$ could have an appreciable value even at $p_{\mathrm{T}} \approx 10 \mathrm{GeV}$ in $0-1 \%$ centrality $5.02 \mathrm{TeV} p+\mathrm{Pb}$ events. Shortly after ISMD19, new results from ATLAS were presented in which jet-triggered events were used in the significantly higher-luminosity $8.16 \mathrm{TeV} p+\mathrm{Pb}$ data taken in 2016 [10]. In these results, shown in Fig. 2, a non-zero azimuthal anisotropy was observed for particles up to $p_{\mathrm{T}} \approx 50 \mathrm{GeV}$, far from the hydrodynamic regime, and over a broad centrality range. The overall shape of the $v_{2}$ as a function of $p_{\mathrm{T}}$ is remarkably similar to that in $\mathrm{A}+\mathrm{A}$ collisions, again suggesting that $\mathrm{A}+\mathrm{A}-$ like effects are present in $p+\mathrm{A}$ collisions. These results exacerbate the already significant tension with a minimum-bias $R_{p \mathrm{~A}}$ factor of unity. 

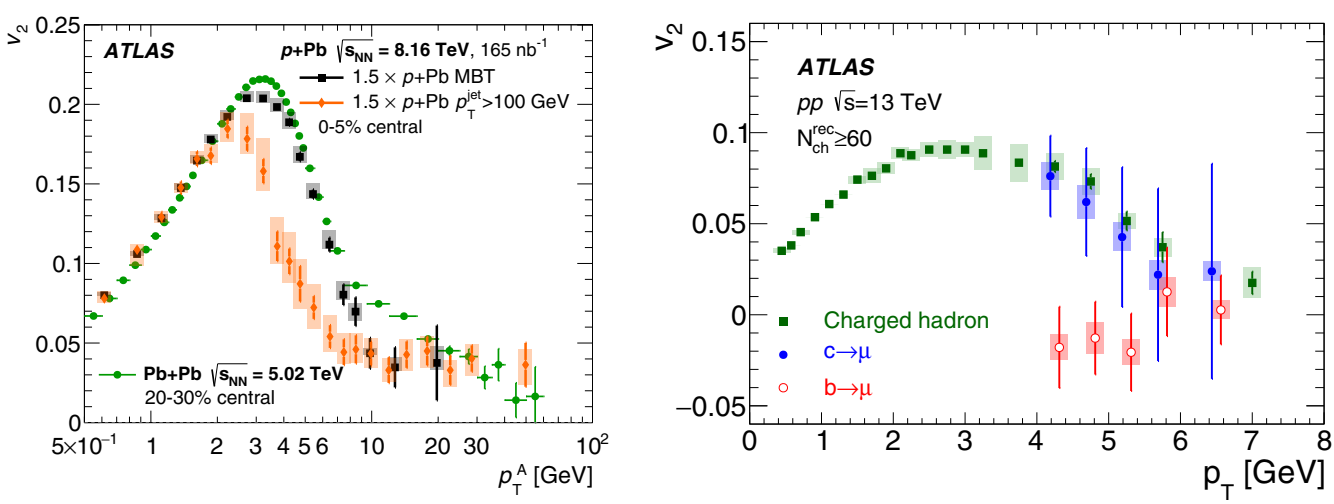

Figure 2. Left: Measured azimuthal anisotropy coefficients $v_{2}$ in $p+\mathrm{Pb}$ collisions using $8.16 \mathrm{TeV}$ data, from Ref. [10]. Right: Measured $v_{2}$ for muons from charm and bottom hadron decays, compared to that for light hadrons, in high-multiplicity $13 \mathrm{TeV} p p$ collisions, from Ref. [11].

\subsection{Jet rates and modification}

Of course, azimuthal anisotropies at high $p_{\mathrm{T}}$ are not the only possible manifestation of $\mathrm{A}+\mathrm{A}$-style partonic energy loss. Following the analogy with A+A collisions, it is useful to have measurements of the nuclear modification factor for various strongly-interacting probes, particularly for system sizes in the transition region from peripheral $\mathrm{A}+\mathrm{A}$ to central $p+\mathrm{A}$, over which energy loss effects are rapidly turning off. Unfortunately, this region is bracketed by the opposite extrema of each type of system, and each is potentially affected by large biases. Central $p+\mathrm{A}$ collisions feature auto-correlations between hard processes and soft particle production $[13,14]$, while selections on very peripheral A + A collisions may suffer from opposite (i.e. jet veto) effects [15]. Model-dependent corrections can be applied to account for these, but it is difficult to obtain the needed level of precision to observe or rule out energy loss effects.

Coincidence measurements where jets are paired with an electroweak boson, another jet, or a high$p_{\mathrm{T}}$ trigger particle can avoid this specific limitation. These measurements are sensitive to energy loss effects which distort the expected kinematic balance between final-state objects, and can be reported as a function of event activity. For example, a measurement by ALICE of semi-inclusive recoil jet distributions in $5.02 \mathrm{TeV}$ data [16] provides constraints on the out-of-cone energy loss. However, this strategy also comes with potential pitfalls: they may have a reduced sensitivity to energy loss (e.g. a di-jet measurement where both jets lose energy, leaving them balanced on average) and the multiplicity selection itself may incur significant biases (e.g. in $p p$ collisions, the resulting enhancement of multi-jet event topologies may significantly modify particular observables). Nevertheless, additional coincidence measurements enabled by the high-statistics $8.16 \mathrm{TeV} p+\mathrm{Pb}$ data would be very valuable.

Finally, the jet-medium interaction in $p+$ A collisions may result only in modest in-cone modification of the parton shower, and thus give a null result in the above approaches. In this case, precision measurements of jet structure in $p+\mathrm{A}$ collisions are needed. The techniques learned from successful measurements A+A collisions [17, 18], along with comparisons to state-of-the-art pQCD calculations such as $\operatorname{SCET}_{G}$ [19], can be applied here. Some initial measurements in this direction of fragmentation functions [20] and jet masses [17] have been performed but only in minimum-bias $p+$ A collisions where jet modification effects are likely to be very modest. 


\subsection{Heavy flavor probes}

Since high- $p_{\mathrm{T}}$ light hadrons are produced as part of the jet fragmentation process, it is possible they are not sensitive to the QGP medium in $p+\mathrm{A}$ collisions. This may occur if there is a finite formation time, or a virtuality separation between the developing shower of the hard-scattered parton and the short-lived medium. This possibility can be avoided with heavy flavor quarks, which due to their large mass must be created early in the collision and thus are present as a conserved flavor charge throughout the full evolution of the medium. Historically, the first measurements of simultaneous flow and suppression of heavy quarks in A+A collisions [21] were important in indicating that a stronglycoupled medium was indeed being formed at RHIC. Studies at the LHC indicate that charm quarks approach equilibrium with the QGP and are thus sensitive to the shape of the produced region [22].

Current data on heavy flavor quark production in $p+\mathrm{A}$ collisions indicates no modification at the $10 \%$ level in production spectra compared to $p p$ collisions [23], except perhaps at very low $p_{\mathrm{T}}$. However, identified $D$-mesons show a significant azimuthal anisotropy in high-multiplicity events over a wide $p_{\mathrm{T}}$ range [6]. The combination of no modification in the $p_{\mathrm{T}}$ distribution with an appreciable modulation results in tension: in a final-state-effect picture, how can the trajectories of the heavy quarks be redirected to such a large degree without a corresponding modification to the $p_{\mathrm{T}}$ distribution?

New ATLAS data on the azimuthal anisotropies of heavy flavor muons bend this contradiction to its breaking point [11], and are summarized in Fig. 2. Whereas charm hadrons are observed to flow with a similar magnitude as light hadrons for a broad range of $p p$ multiplicities, the anisotropy for bottom hadrons is compatible with zero! This is one of the rare strongly interacting probes which is observed to not participate in collective motion ${ }^{1}$. An alternative possibility is that this signal arises from initial-state momentum correlations [25], however this picture has challenges of its own in describing the multiplicity dependence of the data and will not be discussed further here.

These data indicate the special role of heavy flavor quarks in delineating boundaries for final-state collectivity, such as the degree of thermalization or the system size and lifetime. To determine the limiting conditions for $b$-quark flow to appear, a precise measurement in $p+\mathrm{A}$ collisions would be very valuable. Shortly after ISMD19, a measurement of non-prompt $D^{0}$ flow was made by CMS [26], but within the reported uncertainties it is compatible with the level of charm flow, or no flow at all.

\section{Novel collision systems}

If a deconfined QGP medium is indeed created in $p+\mathrm{A}$ and $p p$ collisions, one may expect several possible experimental signatures. For the case of azimuthal anisotropies, the signature primarily focused on here, a sufficiently high multiplicity is not by itself determinative. In the final state interaction picture, it is the underlying transverse spatial geometry of the system which is translated to a momentum anisotropy in the transverse plane. To better test this picture, it is interesting to study exotic collision systems where the transverse geometry is very different and any possible initial-state effects [29] have a different relative contribution. For example, consider the AMPT study described in Ref. [27], which is shown in Fig. 3. If one models a $e^{+} e^{-} \rightarrow q \bar{q}$ event as a single string stretched between the outgoing quarks, even with final-state interactions, no $v_{2}$ is generated since there is no common event-wide geometry. On other hand, the fictitious scenario in which the total energy or string tension is split between two nearby, parallel strings does generate a $v_{2}$.

These kinds of pictures can be tested in data: there have been tremendous efforts to recover old collider data and re-analyze them in the modern context of two- and multi-particle correlation analyses which characterize azimuthally anisotropic particle production. The publically available results so far

\footnotetext{
${ }^{1} \mathrm{~A}$ similarly interesting example is the recent indication that the $v_{2}$ for Upsilon particles - a $b \bar{b}$ bound state - is compatible with zero in $\mathrm{Pb}+\mathrm{Pb}$ collisions [24]. Perhaps the common heavy quark content is not coincidental.
} 

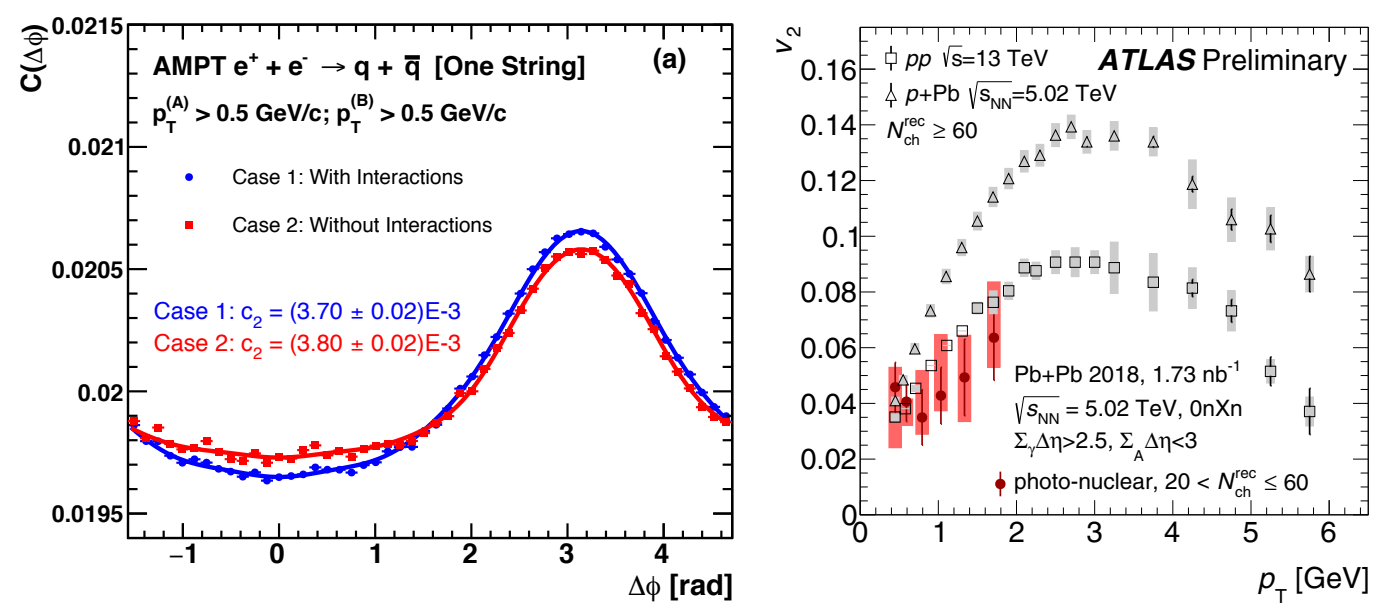

Figure 3. Left: Two-particle $\Delta \phi$ distributed in an AMPT simulation of $e^{+} e^{-} \rightarrow q \bar{q}$ events with a single string between the outgoing quarks, from Ref. [27]. Right: Measured $v_{2}$ in photo-nuclear events, compared to that in $p p$ and $p+\mathrm{A}$ events, from Ref. [28].

are a re-analysis of $91 \mathrm{GeV} e^{+} e^{-}$collisions with ALEPH at LEP [30] and $316 \mathrm{GeV}$ ep collisions with ZEUS at HERA [31], with others underway. In the first measurement, upper statistical limits are set on the possible magnitude of $v_{2}$, while the latter does not observe compatible patterns in the data. Since these experiments and facilities are no longer operating, it is difficult to imagine significant improvements in the archived data, and insight may come only from future data on novel collision systems at RHIC, the LHC, and eventually the Electron Ion Collider (EIC).

Another interesting possibility is to explore the so-called ultra-peripheral A+A processes, electromagnetic interactions in which the nuclear beams remain separated. Among these, photo-nuclear $(\gamma+\mathrm{A})$ interactions are those in which one nucleus emits a quasi-real photon which strikes the other nucleus. These events feature a significantly cleaner environment for exploring nuclear effects than $p+$ A collisions and allow for the possibility of accessing "nuclear-DIS-like" physics (at least, at the photoproduction limit of DIS) before the EIC is operating.

These events can also be used to search for collective phenomena, such as the analysis of highmultiplicity photo-nuclear events in Ref. [28], shown in Fig. 3. Using standard two-particle correlation methods, a symmetric ridge can be observed in these events. When interpreted as a single-particle anisotropy under the assumptions of factorization and the non-flow subtraction procedure, it results in $v_{2}$ values compatible with that in $p p$ collisions but lower than that in $p+\mathrm{Pb}$. However, this may not be surprising in a final-state picture: in the vector-meson dominance (VMD) paradigm, the photon may fluctuate to a meson, especially under a high multiplicity selection. Thus many of these collisions may proceed as $\rho+\mathrm{A}$ or $\omega+\mathrm{A}$ collisions with a non-trivial transverse geometric structure which may induce momentum-space anisotropies. A more quantitative comparison to theory which matches the particular $\gamma+$ A kinematics and experimental acceptance would be very interesting.

\section{Conclusion}

To better understand the connection between soft and hard probes of small collision systems, several specific approaches can be proposed. Precision jet-coincidence and jet-structure measurements 
should continue to be performed in $p+$ A collisions, ideally with guidance from theoretical approaches that correctly describe A+A collisions. Measurements of azimuthal anisotropy should be extended to higher $p_{\mathrm{T}}$ and should particularly be performed for charm and bottom quarks as key discriminants. New collision systems at RHIC and the LHC, such as possible $\mathrm{O}+\mathrm{O}$ or $\mathrm{Ar}+\mathrm{Ar}$ running [32], are important for filling in the $p+\mathrm{A} / \mathrm{A}+\mathrm{A}$ "gap" without biases arising from extreme multiplicity selections. Finally, novel systems such as those accessible in ultra-peripheral collisions and at the future EIC will be important for testing the final-state interaction picture in situations with starkly different geometries.

\section{References}

[1] J.L. Nagle, W.A. Zajc, Ann. Rev. Nucl. Part. Sci. 68, 211 (2018), 1801.03477

[2] J. Adolfsson et al. (2020), 2003. 10997

[3] R.D. Weller, P. Romatschke, Phys. Lett. B 774, 351 (2017), 1701. 07145

[4] C. Aidala et al. (PHENIX), Nature Phys. 15, 214 (2019), 1805.02973

[5] P. Romatschke, Eur. Phys. J. C 78, 636 (2018), 1802 . 06804

[6] A.M. Sirunyan et al. (CMS), Phys. Rev. Lett. 121, 082301 (2018), 1804.09767

[7] C. Aidala et al. (PHENIX), Phys. Rev. Lett. 120, 062302 (2018), 1707.06108

[8] J. Noronha-Hostler et al., Phys. Rev. Lett. 116, 252301 (2016), 1602 . 03788

[9] X. Zhang, J. Liao (2013), 1311. 5463

[10] G. Aad et al. (ATLAS), Eur. Phys. J. C 80, 73 (2020), 1910. 13978

[11] G. Aad et al. (ATLAS), Phys. Rev. Lett. 124, 082301 (2020), 1909.01650

[12] G. Aad et al. (ATLAS), Phys. Rev. C 90, 044906 (2014), 1409. 1792

[13] J. Adam et al. (ALICE), Phys. Rev. C 91, 064905 (2015), 1412.6828

[14] D.V. Perepelitsa, P.A. Steinberg (2014), 1412.0976

[15] C. Loizides, A. Morsch, Phys. Lett. B 773, 408 (2017), 1705.08856

[16] S. Acharya et al. (ALICE), Phys. Lett. B 783, 95 (2018), 1712.05603

[17] S. Acharya et al. (ALICE), Phys. Lett. B 776, 249 (2018), 1702.00804

[18] M. Aaboud et al. (ATLAS), Phys. Rev. Lett. 123, 042001 (2019), 1902 . 10007

[19] Z.B. Kang et al., Phys. Rev. Lett. 114, 092002 (2015), 1405 . 2612

[20] M. Aaboud et al. (ATLAS), Nucl. Phys. A 978, 65 (2018), 1706.02859

[21] A. Adare et al. (PHENIX), Phys. Rev. Lett. 98, 172301 (2007), nucl-ex/0611018

[22] S. Acharya et al. (ALICE), JHEP 02, 150 (2019), 1809. 09371

[23] J. Adam et al. (ALICE), Phys. Rev. C 94, 054908 (2016), 1605.07569

[24] S. Acharya et al. (ALICE), Phys. Rev. Lett. 123, 192301 (2019), 1907.03169

[25] C. Zhang et al., Phys. Rev. Lett. 122, 172302 (2019), 1901.10320

[26] CMS Collaboration, CMS-PAS-HIN-19-009 (2019)

[27] J. Nagle et al., Phys. Rev. C 97, 024909 (2018), 1707.02307

[28] ATLAS Collaboration, ATLAS-CONF-2019-022 (2019)

[29] B. Schenke, C. Shen, P. Tribedy, Phys. Lett. B 803, 135322 (2020), 1908.06212

[30] A. Badea et al., Phys. Rev. Lett. 123, 212002 (2019), 1906.00489

[31] I. Abt et al. (ZEUS), JHEP 04, 070 (2020), 1912 . 07431

[32] Z. Citron et al., CERN Yellow Rep. Monogr. 7, 1159 (2019), 1812.06772 